\title{
Self-trapped exciton state in Si nanocrystals revealed by induced absorption
}

\author{
W. D. A. M. de Boer, ${ }^{*}$ D. Timmerman, and T. Gregorkiewicz \\ Van der Waals-Zeeman Institute, University of Amsterdam, Science Park 904, 1098 XH Amsterdam, The Netherlands
}

\author{
H. Zhang and W. J. Buma \\ Van 't Hoff Institute for Molecular Science, University of Amsterdam, Science Park 904, 1098 XH Amsterdam, The Netherlands \\ A. N. Poddubny, A. A. Prokofiev, and I. N. Yassievich \\ Ioffe Physical-Technical Institute, Russian Academy of Sciences and Polytechnicheskaya 26, 194021 Saint-Petersburg, Russia
}

(Received 4 December 2011; revised manuscript received 22 March 2012; published 25 April 2012)

\begin{abstract}
We report results of time-resolved induced absorption (IA) spectroscopy on Si nanocrystals (Si NCs) embedded in $\mathrm{SiO}_{2}$ matrix. In line with theoretical modeling, the IA amplitude decreases with probing photon energy, however only until a certain threshold value. For larger photon energies, an increase of IA is observed. This unexpected behavior is interpreted in terms of the self-trapped exciton state whose formation in Si NCs was put forward some time ago based on theoretical considerations. Here, we present a direct experimental confirmation of this supposition.
\end{abstract}

Silicon nanocrystals $(\mathrm{NCs})^{1}$ are frequently investigated for their interesting optical properties and a wide variety of potential applications in optoelectronics, ${ }^{2-4}$ photovoltaics, ${ }^{5-7}$ and the medical field. ${ }^{8}$ In particular, the photoluminescence (PL) of $\mathrm{Si} \mathrm{NCs}$ has been thoroughly characterized by experiment ${ }^{9,10}$ and extensively modeled by theory using the $a b$ initio approach ${ }^{11}$ as well as the semiempirical methods: pseudopotential, ${ }^{12}$ tight-binding, ${ }^{13,14}$ and effective mass approximation. ${ }^{15}$ In that way, opening of the (indirect) band gap and enhancement of the radiative rate of band-to-band recombination have been firmly established. For oxygenterminated Si NCs a peculiarity has been found: for smaller diameters $d_{N C}<2.5 \mathrm{~nm}$ the blueshift in PL spectrum could not be observed, with PL energy stabilizing in the visible range. This has been explained in terms of formation of oxygenrelated defects at the surface of NCs, with levels appearing in the band gap and participating in the recombination of carriers. ${ }^{16}$ Specific microscopic details of these defects are not known, but oxygen is well known to form electrically active defects in bulk Si. ${ }^{17,18}$ Among other possibilities, formation of a self-trapped exciton state (STE) has been proposed. ${ }^{19}$ Support for the existence of the STE state facilitating photon emission in small oxygen-terminated Si NCs was inferred only indirectly from steady-state PL experiments-predominantly from the aforementioned stabilization of the quantum confinement induced blueshift of PL and from the temperature dependence of PL intensity and lifetime. ${ }^{20}$ It is fair to say that an experimental evidence directly confirming formation of the STE is still missing.

In order to investigate formation and characteristics of the STE state, carrier dynamics directly after photoexcitation need to be investigated. This is best accomplished by means of ultrafast induced absorption (IA) and PL up-conversion spectroscopy. Past investigations revealed that carrier dynamics in Si NCs exhibits always a fast multiexponential decay. ${ }^{21-24}$ This illustrates a variety of carrier relaxation pathways, with individual components assigned to trapping, ${ }^{25}$ carrier-carrier scattering, Auger energy transfer between electrons and holes ${ }^{14}$ phonon-assisted cooling, and no-phonon radiative recombination. ${ }^{10}$ In that landscape, formation of the STE state has been related to trapping to defects at the surface of $\mathrm{Si}$ NCs, competing with carrier cooling. For oxygen-passivated Si NCs, it has been shown that effective carrier cooling can be slowed down by three orders of magnitude in comparison to bulk $\mathrm{Si},{ }^{10}$ making trapping processes even more efficient.

In this study, we present conclusive evidence for formation of a STE in Si NCs in an oxygen-rich environment. These NCs are characterized by the typical excitonic emission, blueshifting for smaller sizes. Formation of the STE state has been investigated with ultrafast IA performed over a broad probing range (1.6-3.25 eV). Direct evidence of the STE state is obtained from the time-resolved spectral characteristics of the IA.

The study has been performed on $\mathrm{Si}$ NCs embedded in a $\mathrm{SiO}_{2}$ matrix, which were prepared by a radio-frequency co-sputtering method. By tuning excess of $\mathrm{Si}$ and annealing temperature, average $\mathrm{NC}$ sizes in the $d_{N C}=2.5-5 \mathrm{~nm}$ range were obtained in the $\sim 1-\mu \mathrm{m}$-thick optically active layer. ${ }^{26}$ For IA experiments, a pump-probe setup was employed. Primary pulse is provided by an optical parametric amplifier, pumped by an amplified Ti:Sapphire laser, with a repetition rate of $f=$ $1 \mathrm{kHz}$ (resolution $\sim 200 \mathrm{fs}$ ). The probe pulse was converted to a broad-band white light continuum $\left(E_{\text {probe }}=1.6-3.25 \mathrm{eV}\right)$, in order to enable energy-resolved probing. Optical delay lines were folded to enlarge the detection time window to several ns. IA signal was detected with a multichannel CCD camera, and registered as $I_{I A}=\left(I_{\text {total }}-I_{\text {lin.abs }}\right) / I_{\text {lin.abs. }}$. Here $I_{I A}$ is the IA signal, $I_{\text {total }}$ the combined linear and induced absorption of the probe pulse, and $I_{\text {lin.abs }}$ is the linear absorption, which is subtracted to separate the contribution of the free carrier absorption. The IA signal is normalized to the linear absorption of the probe; in this way, the relative change of the IA with respect to the linear absorption is obtained, and the effect of pump power fluctuations is eliminated. The baseline of the IA dynamics is established by tuning the probe pulse prior to the excitation pulse. All experiments were performed under the 


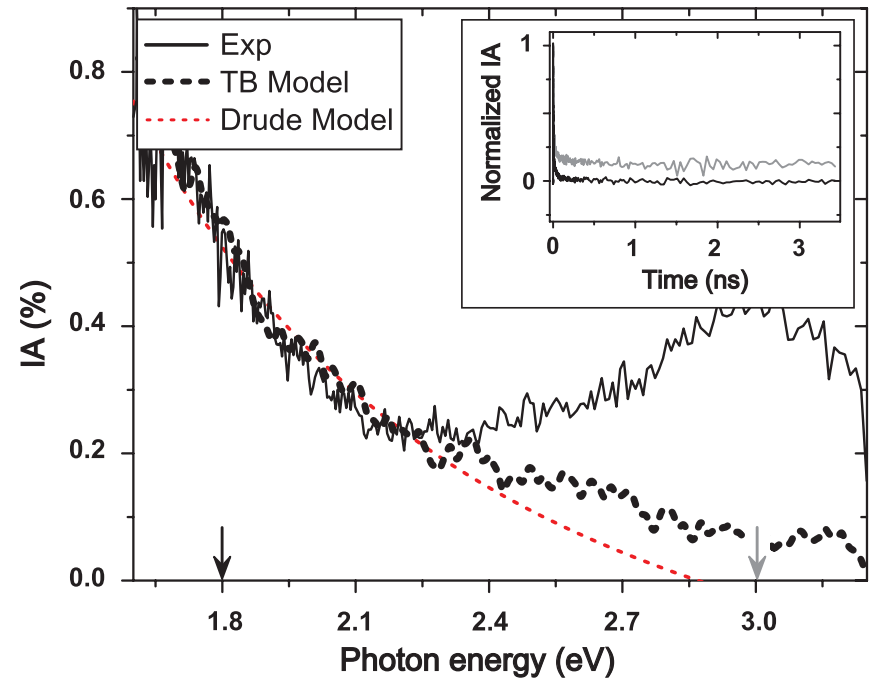

FIG. 1. (Color online) IA spectra and dynamics in the visible probing regime $\left(E_{\text {probe }}=1.6-3.25 \mathrm{eV}\right)$ for the sample with average diameter $d_{N C}=4 \mathrm{~nm}$ under excitation of $E_{\text {exc }}=3.6 \mathrm{eV}$ at maximum temporal overlap between pump and probe pulses. The decrease in IA intensity for small photon energies is followed by an increase in the high photon energy range, starting from the threshold value $E_{\text {th }} \approx$ $2.4 \mathrm{eV}$. (The decrease in IA intensity at the edges of the presented regime is a result of experimental detection limitations.) The two arrows indicate the photon energies for which transients are displayed in the inset. The dashed curves show the simulations of the probing photon energy dependent IA signal obtained by the semi-empirical $s p^{3} d^{5} s^{*}$ tight-binding (black) and Drude (red dashed) model. Inset: (Normalized) IA transient for $E_{\text {probe }}=1.8 \mathrm{eV}$ (black) $E_{\text {probe }}=3 \mathrm{eV}$ (gray) in a $3.5 \mathrm{~ns}$ time window, with initial fast decay components (1-100 ps) and a component extending to the ns- $\mu$ s-range for the high photon energy excitation.

excitation condition of multiple excitons per $\mathrm{NC}$ and at room temperature.

For all the samples investigated in this study, we have found IA spectra to be independent of excitation photon energy [ranging from $E_{\mathrm{exc}}=2.5-3.8 \mathrm{eV}$ (not shown)]. This provides direct evidence that the IA cross section of photo-generated carriers is practically independent of their energies. In Fig. 1, the IA spectrum for the sample with average NC diameter of $d_{N C}=4 \mathrm{~nm}$ is shown, as obtained under excitation at $E_{\text {exc }}=$ $3.6 \mathrm{eV}$ for the maximum temporal overlap between pump and probe pulses. The experimental results are compared to two curves obtained by different theoretical approaches for the IA cross section which give similar structural dependence: the Drude model, with a $\lambda^{-2}$-dependence ${ }^{27}$ (red dashed curve), and semiempirical $s p^{3} d^{5} s^{*}$ tight-binding calculations (black dashed curve). ${ }^{28,29}$ Both models predict that the amplitude of the IA signal should decrease gradually toward higher probing photon energies. This is indeed observed in the experiment for low detection photon energies, up to the threshold value $E_{\mathrm{th}} \approx 2.4 \mathrm{eV}$. For photon energies exceeding this threshold, the experimental results diverge from theoretical modeling, with the measured IA spectrum increasing with probing photon energy. The inset of Fig. 1 displays IA transients recorded for two probing photon energies, below $\left[E_{\text {probe }}=1.8 \mathrm{eV}\right.$ (black)] and above $\left[E_{\text {probe }}=3 \mathrm{eV}\right.$ (gray)] the threshold energy, indicated by the arrows in the main panel. Both traces feature a multiexponential decay, with characteristic time constants of the order of 1-100 ps. For the low probing photon energy, the IA signal practically vanishes within $1 \mathrm{~ns}$. In contrast, the transient taken for $E_{\text {probe }}=3 \mathrm{eV}$ also shows a much slower background. Since the IA signal is practically stable within the measured time window, we conclude that the "slow" component corresponds to a decay process taking place on the nanosecond to microsecond time scale.

Generally, IA signal reflects concentration of carriers generated by the excitation pulse, where different components in the decay dynamics correspond to various relaxation and/or recombination processes. Since the experiment in this study is conducted under high photon flux, such that multiple carriers are created per single NC, the measured dynamics are dominated by Auger recombination of multiple excitons confined in the same $\mathrm{NC}$, which is known to proceed on a picosecond time scale. ${ }^{10}$ As shown in the inset of Fig. 1, in case of low probing photon energies, the IA signal practically decays entirely on the sub-ns time scale (as far as can be concluded within the experimental resolution), which can be interpreted as total depletion of free carriers. However, this conclusion is contradicted by the second trace in the inset, which shows that free carriers (excitons) are still present when probing is carried out at higher photon energies, above the threshold. Moreover, total depletion of carriers on the fast nanosecond time scale is in direct contradiction with PL results concerning band-to-band radiative recombination. The Si NC samples investigated in this study are characterized by a PL spectrum blueshifting for smaller NC sizes and decaying within $10^{-6}-10^{-4} \mathrm{~s}^{30}$ (in agreement with theoretical modeling for $\mathrm{O}$-passivated $\mathrm{Si} \mathrm{NCs}$ in this size regime ${ }^{31}$ ). Excitons populating the NC-core related levels and undergoing radiative recombination must also be available for probing. At the same time, modeling of the IA cross section within the semiempirical tight-binding approach showed its practical independence on exciton energy, as has also been found in experiments. $^{28}$ Therefore, the decay of IA signal cannot be related to relaxation of free carriers toward lower energy states, and a different explanation must be sought for.

The fact that for long delay times between pump and probe $(\Delta t \gtrsim 0.5 \mathrm{~ns})$ carriers are not available for detection by photons in the lower energy range of the probe spectrum, and are "visible" only for photons whose energy exceeds a certain threshold, suggests efficient trapping of free carriers. The initial rapid decrease of absorption observed at all probing energies then reflects reduction of free carrier concentration due to efficient trapping. The nature of the trap state has to fulfill two conditions: the trapped carriers (i) need to be able to return to the free exciton (FE) state from where they can recombine radiatively, and (ii) need to be available for probing with photons of sufficient energy $E_{\text {probe }}>E_{\text {th }}$. This suggests that carriers are not captured at a charge-mediated trap state, but rather by means of dipole-dipole interaction between exciton and surface state, or by Coulombic interaction between hole and electron, with one of them initially being localized at the surface state. With such a STE scenario, absorption for the low photon energy range vanishes, but remains possible for 


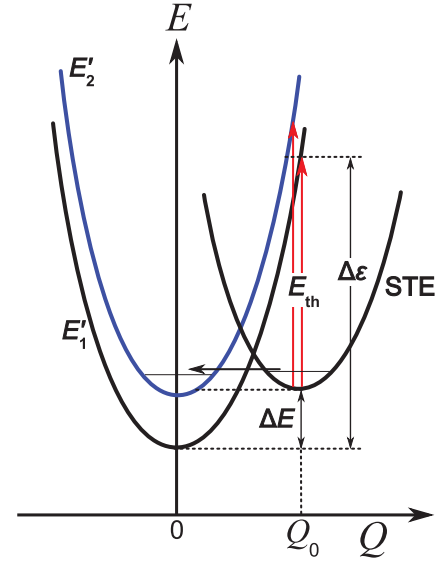

FIG. 2. (Color online) Configuration coordinate diagram with adiabatic potentials for $\mathrm{FE}\left(E^{\prime}\right)$, and STE. Black $\left(E_{1}^{\prime}\right)$ and blue parabolas $\left(E_{2}^{\prime}\right)$ represent FE state for "large" and "small" NC sizes, respectively. The minimum of the parabola for the STE is shifted to $Q_{0}$ compared to the FE adiabatic potential. The red arrows correspond to the energy required for optical re-excitation from the STE to the FE state- $E_{\mathrm{th}}$. This can also be achieved by thermally stimulated tunneling ionization - shown by the black horizontal arrow. $\Delta E$ corresponds to the energy difference between the bottom of the FE and the STE adiabatic potentials, and $\Delta \epsilon$ to the sum of $\Delta E$ and $E_{\mathrm{th}}$, respectively.

above-threshold energies, for as long as carriers are trapped in the STE state.

A qualitative description of the STE scenario can be achieved in the Huang-Rhys model within a configuration coordinate diagram (see Fig. 2 for a schematic illustration). Here the adiabatic potentials correspond to the exciton state of $\mathrm{NC}$ in the ground state $\left(E^{\prime}\right)$ and the exciton trapped at a defect state (STE). The potentials depend on the displacement coordinate $Q$, where the position of the minimum of the STE parabola $Q_{0}$ represents the exciton-phonon interaction strength. ${ }^{32,33}$ Generally, it can be assumed that the energy position of STE states is marginally dependent on NC size. ${ }^{16}$ On the other hand, the FE adiabatic potential shifts up in energy when NC size is reduced, as a result of opening of the $\mathrm{NC}$ band gap. This is illustrated by the two parabolas, where $E_{1}^{\prime}$ represents the adiabatic potential for a larger NC (black) and $E_{2}^{\prime}$ for a smaller one (blue). The minimal energy required to release the carriers from the STE state into a FE by means of photon absorption $E_{\mathrm{th}}$ is indicated by the red arrows. It should be noted that carriers which return or remain in the FE state should also be available for probing with high photon energies, although their contribution to the IA signal will be significantly less, as a consequence of smaller IA cross section (see Fig. 1). As can be seen, the energy required to release an exciton from the self-trapped state increases for smaller NCs due to quantum confinement. In order to validate this scenario, IA spectra for Si NCs of different sizes need to be compared, so that the NC size dependence of the threshold energy $E_{\mathrm{th}}$ can be established.

Figure 3(a) displays IA spectra obtained for Si NC with average diameters of $d_{N C}=2.5 \mathrm{~nm}$ (black), $4 \mathrm{~nm}$ (green), and $5 \mathrm{~nm}$ (red) for a long delay time between pump and probe pulse ( $\Delta t \approx 1 \mathrm{~ns}$ ). Within the STE scenario, after such a time interval carrier trapping should be completed and absorption of probe can proceed practically exclusively by optical ionization of the STE state. Indeed, a clear shift of the threshold energy marking higher absorption toward higher photon energies is observed: For the largest NC size $E_{\mathrm{th}} \approx 2.25 \mathrm{eV}$ is found, shifting to $E_{\text {th }} \approx 2.45 \mathrm{eV}$ for $d_{N C}=4 \mathrm{~nm}$ and to $E_{\text {th }} \approx 2.75 \mathrm{eV}$ for the 2.5-nm-sized NCs. The value of this blueshift-about $0.5 \mathrm{eV}$ for the investigated size range-agrees reasonably well with the blueshift of the indirect bandgap $(\sim 0.45 \mathrm{eV})$, concluded from the excitonic PL for the same materials ${ }^{10}$ [illustrated in Fig. 3(b), showing the maximum of the PL spectrum of each sample]. This similarity evidences the aforementioned practical independence of the STE energy position on NC size. Since PL should be dominated by band-to-band recombination from the lower lying FE state, we can assume that in that case the STE state is metastable, with its energy being positioned (at least) $\Delta E \approx 0.5 \mathrm{eV}$ above the FE ground state of Si NCs with $d_{N C}=5 \mathrm{~nm}$. This value agrees reasonably well with the one found in Ref. 16, taking into account the energy shift due to Coulombic interaction for exciton. ${ }^{15,34}$ This results in the binding energy of STE $\Delta \varepsilon=\Delta E+E_{\text {th }} \approx 2.75 \mathrm{eV}$-see Fig. 2 .

Following the proposed model, the decay dynamics for the above-threshold absorption will reflect the STE lifetime and will correspond to the slow component of IA in the high photon energy regime-see the transient (gray) in the inset to Fig. 1. The STE lifetime could be controlled by direct recombination (radiative and nonradiative) and/or by thermally stimulated tunneling ionization. The probability of the latter process is $W_{t} \equiv\left(\tau_{t}\right)^{-1}=w_{e} J\left(S_{H R}, T, p\right)$, where $w_{e}$ is the pure electron transition probability and $J\left(S_{H R}, T, p\right)$ is the overlap integral of oscillator wave functions which depends on temperature $T$, the Huang-Rhys factor $S_{H R}=\Delta \varepsilon / \hbar \omega_{0}$, and the parameter $p \approx \Delta E / \hbar \omega_{0}$, which is the number of phonons involved in the transition. The precise expression for $J\left(S_{H R}, T, p\right)$ was obtained by Huang and Rhys. ${ }^{35}$ In the first approximation, $w_{e}$ is independent of the vibration coordinate, and can be treated as a parameter. For the phonon we take the $\mathrm{Si}-\mathrm{O}$ vibrational mode $\hbar \omega_{0}=140 \mathrm{meV},{ }^{36}$ resulting in the Huang-Rhys factor $S_{H R} \approx 20.1$. We note that this value is similarly large as obtained in the past for the DX center in AlGaSb:Te. ${ }^{37}$ The value of $w_{e}$ can be estimated from the capture probability $W_{c}$; according to our model this can be extracted from the initial IA dynamics for small probing energy. Since it can be concluded from experiments that trapping time does not dramatically depend on NC size, we use an average value of $\tau_{\mathrm{c}}=10^{-12}-10^{-11} \mathrm{~s}^{38}$ Following the same approximation, we determine the overlap integral for capturing of hot carriers, using excitation energy $E_{\text {exc }}=3.6 \mathrm{eV}$ for which we get $W_{c}=w_{e} \cdot 2 \times 10^{-2} \mathrm{~s}^{-1}$. For the NC sizes used in this study, we find an average value of $w_{e}=10^{13}-10^{14} \mathrm{~s}^{-1}$. The overlap integral for thermally activated tunneling from the STE state to the NC core-related levels for Si NCs with $d_{N C}=5 \mathrm{~nm}$ is $J=1.5 \times 10^{-5}$ at room temperature, yielding the STE lifetime of $\tau_{t}=1.2 \times\left(10^{-9}-10^{-8}\right) \mathrm{s}$. This can be observed as the decay of the tail in IA decay dynamics recorded at $E_{\text {probe }}=$ $3 \mathrm{eV}$ for the 5-nm-sized sample [Fig. 3(c)]. Although accuracy with which the "slow" component can be determined is limited by the fairly low signal-to-noise ratio and the time window being relatively short compared to the expected tunneling time, the value $\tau_{\text {tail }} \approx 5.2 \times 10^{-9} \mathrm{~s}$ extracted from fitting is 

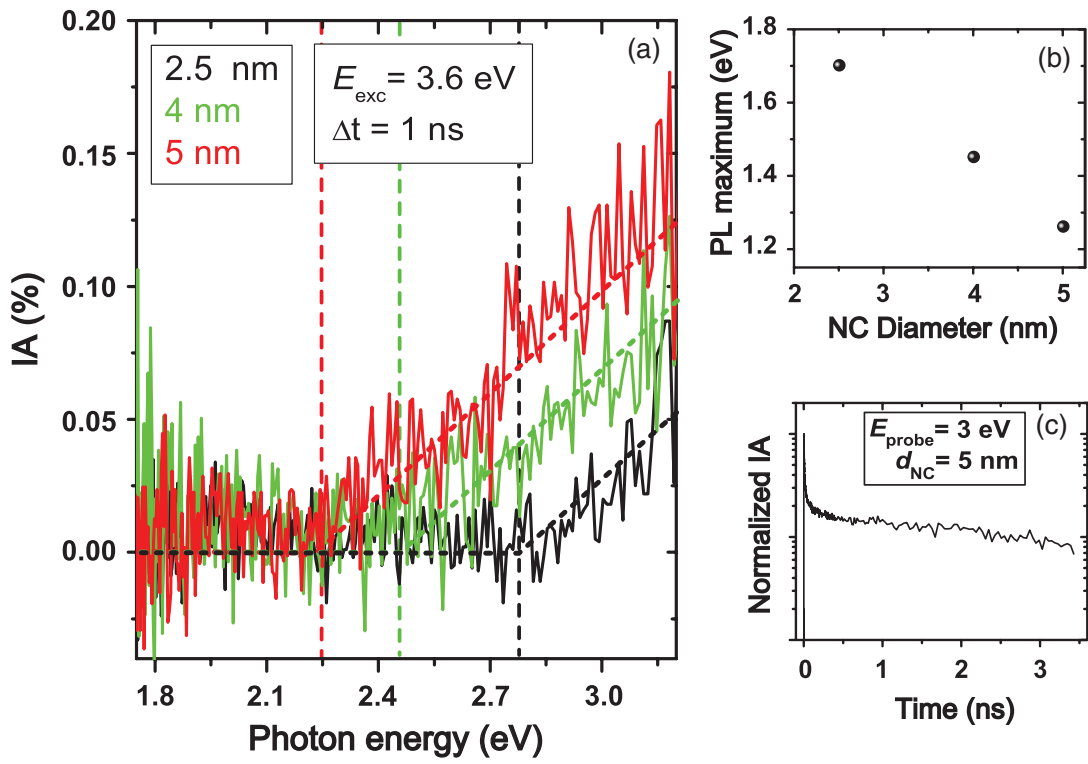

FIG. 3. (Color online) IA en PL results for samples with different average NC sizes. a) IA spectra in the visible regime recorded for $1 \mathrm{~ns}$ delay time between pump and probe pulse for different average sizes of NCs, $d_{N C}=2.5 \mathrm{~nm}$ (black), $4 \mathrm{~nm}$ (green/light gray) and $5 \mathrm{~nm}$ (red/gray), respectively. The threshold value $E_{\text {th }}$ shifts from $E_{\text {th }} \approx$ $2.25 \mathrm{eV}$ for the $5 \mathrm{~nm}$-sized $\mathrm{NC}$ to $E_{\mathrm{th}} \approx$ $2.45 \mathrm{eV}$ for the $4 \mathrm{~nm}$-sized $\mathrm{NC}$, and $E_{\mathrm{th}} \approx$ $2.75 \mathrm{eV}$ for the smallest $\mathrm{NC}$ size used in this study. Similar data have been obtained for NCs of the same size, prepared by different-not shown b) Maximum of the PL spectrum for the same samples as presented in panel (a). c) IA transient for the $5 \mathrm{~nm}$-sized NCs recorded at $E_{\text {exc }}=$ $3 \mathrm{eV}$. In addition to fast components ranging from $1-100 \mathrm{ps}$, also a ns component is found. in good agreement with the theoretically predicted one. Using the same value for the Huang-Rhys parameter for the other $\mathrm{Si}$ NCs investigated in this study, we get $\tau_{t}=3.3 \times\left(10^{-8}-10^{-7}\right)$ $\mathrm{s}$ and $\tau_{t}=3.4 \times\left(10^{-6}-10^{-5}\right) \mathrm{s}$ for $d_{N C}=4 \mathrm{~nm}$ and $d_{N C}=$ $2.5 \mathrm{~nm}$, respectively. The increase of STE lifetime for smaller sizes is demonstrated in the inset of Fig. 1, showing absence of a component decaying on the ns timescale in the transient recorded at high photon energy (gray) for the 4-nm-sized NCs. Moreover, according to the Huang-Rhys model the STE lifetime should be dependent on temperature, and is expected to decrease significantly for lower temperatures; this aspect is currently under investigation. For NCs with diameters $d_{N C} \lesssim$ $2.5 \mathrm{~nm}$, the space quantization induced shift of the FE state exceeds that of the STE, i.e., we arrive to a stable STE state. In this case, PL would be realized by recombination from this state rather than from the confined free exciton state, which corresponds to the well-documented stabilization of PL energy for small NC clusters. ${ }^{16}$

The IA data obtained in this study can be fully rationalized within the STE model. Together with the previously reported PL results for different NC sizes, they support the model proposed in the past on the basis of theoretical arguments and supported indirectly by experiment. From the results presented in the current study, we are able to conclude on the formation of STEs directly from the (fast) disappearance of IA signal for low detection photon energies in combination with the long-living absorption for photon energies $h v \gtrsim E_{\mathrm{th}}$. The NC size-dependent shift of the threshold energy $E_{\text {th }}$ necessary for optical ionization of STE represents an experimental fingerprint of this state. The observed blueshift of $E_{\text {th }}$ for smaller NC sizes directly confirms the STE model. In addition, this experiment gives us new insights on the characteristics of the STE state, since the efficiency of the trapping process responsible for formation of STE is directly reflected in IA dynamics.

In conclusion, the presented IA study of $\mathrm{Si}$ NCs embedded in $\mathrm{SiO}_{2}$ matrix provides direct experimental evidence for the formation of the STE state. We show that this state is formed by efficient trapping of free carriers on a subnanosecond scale. Once trapped, the excitons can be released by optical absorption with photons of sufficiently large energy, or by thermal activation. Since the defect level determining the energy of STE is independent of NC size while the band-gap energy changes due to quantum confinement, the energy barriers for both processes increase for smaller nanocrystals. Consequently, formation of STE does not influence emission for large Si NCs for which the STE energy exceeds the band gap, and the thermally activated release is efficient. The situation changes for small NCs, with the STE state becoming more stable, and therefore dominating PL. *w.d.a.m.deboer@uva.nl

${ }^{1}$ L. Canham, Appl. Phys. Lett. 57, 1046 (1990).

${ }^{2}$ L. Pavesi, L. Dal Negro, C. Mazzoleni, G. Franzò, and F. Priolo, Nature (London) 408, 440 (2000).

${ }^{3}$ I. Izeddin, D. Timmerman, T. Gregorkiewicz, A. S. Moskalenko, A. A. Prokofiev, I. N. Yassievich, and M. Fujii, Phys. Rev. B 78, 035327 (2008).

${ }^{4}$ K. Dohnalová, K. Žídek, L. Ondič, K. Küsová, O. Cibulka, and I. Pelant, J. Phys. D: Appl. Phys. 42, 135102 (2009).
${ }^{5}$ D. Timmerman, I. Izeddin, P. Stallinga, I. N. Yassievich, and T. Gregorkiewicz, Nature Phot. 2, 105 (2008).

${ }^{6}$ D. Timmerman, J. Valenta, K. Dohnalová, W. D. A. M. De Boer, and T. Gregorkiewicz, Nature Nanotech. 6, 710 (2011).

${ }^{7}$ W. de Boer and T. Gregorkiewicz, SPIE Newsroom (2012), doi:10.1117/2.1201202.004136.

${ }^{8}$ J.-H. Park, L. Gu, G. von Maltzahn, E. Ruoslahti, S. N. Bhatia, and M. J. Sailor, Nat. Mater. 8, 331 (2009).

${ }^{9}$ D. Kovalev H. Heckler, G. Polisski, and F. Koch, Phys. Stat. Sol. 215, 871 (1999). 
${ }^{10}$ W. D. A. M. de Boer, D. Timmerman, K. Dohnalová, I. N. Yassievich, H. Zhang, W. J. Buma, and T. Gregorkiewicz, Nature Nanotech. 5, 878 (2010).

${ }^{11}$ E. Degoli, G. Cantele, E. Luppi, R. Magri, D. Ninno, O. Bisi, and S. Ossicini, Phys. Rev. B 69, 155411 (2004).

${ }^{12}$ C. Bulutay, Phys. Rev. B 76, 205321 (2007).

${ }^{13} \mathrm{C}$. Delerue and M. Lannoo, Nanostructures: Theory and Modelling (Springer-Verlag, Berlin, 2004).

${ }^{14}$ A. N. Poddubny, A. A. Prokofiev, and I. N. Yassievich, Appl. Phys. Lett. 97, 231116 (2010).

${ }^{15}$ A. S. Moskalenko, J. Berakdar, A. A. Prokofiev, and I. N. Yassievich, Phys. Rev. B 76, 085427 (2007).

${ }^{16}$ M. V. Wolkin, J. Jorne, P. M. Fauchet, G. Allan, and C. Delerue, Phys. Rev. Lett. 82, 197 (1999).

${ }^{17}$ H. H. P. Th. Bekman, T. Gregorkiewicz, D. A. van Wezep, and C. A. J. Ammerlaan, J. Appl. Phys. 62, 4404 (1987).

${ }^{18}$ T. Gregorkiewicz, D. A. van Wezep, H. H. P. Th. Bekman, and C. A. J. Ammerlaan, Phys. Rev. Lett. 59, 1702 (1987).

${ }^{19}$ G. Allan, C. Delerue, and M. Lannoo, Phys. Rev. Lett. 76, 2961 (1996).

${ }^{20}$ K. S. Zhuravlev, and A. Yu. Kobitsky, Semiconductors 34, 1203 (2000).

${ }^{21}$ W. D. A. M. de Boer, M. T. Trinh, D. Timmerman, J. M. Schins, L. D. A. Siebbeles, and T. Gregorkiewicz, Appl. Phys. Lett. 99, 053126 (2011).

${ }^{22}$ E. Lioudakis, A. Othonos, and A. G. Nassiopoulou, Appl. Phys. Lett. 90, 171103 (2007).

${ }^{23}$ M. Beard, K. P. Knutsen, Y. Pingrong, J. M. Luther, Q. Song, W. K. Metzger, R. J. Ellingson, and A. J. Nozik, Nano Lett. 7, 2506 (2007).
${ }^{24}$ V. I. Klimov, Ch. J. Schwarz, D. W. McBranch, and C. W. White, Appl. Phys. Lett. 73, 2603 (1998).

${ }^{25}$ F. Trojánek, K. Neudert, M. Bittner, and P. Malý, Phys. Rev. B 72, 075365 (2005).

${ }^{26}$ S. Takeoka, M. Fujii, and S. Hayashi, Phys. Rev. B 62, 16820 (2000).

${ }^{27}$ R. D. Kekatpure and M. L. Brongersma, Nano Lett. 8, 3787 (2008).

${ }^{28}$ W. D. A. M. De Boer, D. Timmerman, A. N. Poddubny, A. A. Prokofiev, I. N. Yassievich, H. Zhang, W. J. Buma, and T. Gregorkiewicz (to be submitted).

${ }^{29}$ J.-M. Jancu, R. Scholz, F. Beltram, and F. Bassani, Phys. Rev. B 57, 6493 (1998).

${ }^{30}$ D. Timmerman, I. Izeddin, and T. Gregorkiewicz, Phys. Status Solidi A 207, 183 (2010)

${ }^{31}$ M. S. Hybertsen, Phys. Rev. Lett. 72, 1514 (1994).

${ }^{32}$ V. N. Abakumov, V. I. Perel, and I. N. Yassievich, Nonradiative Recombination in Semiconductors (North-Holland, Amsterdam, 1991).

${ }^{33}$ A. N. Poddubny and S. V. Goupalov, Phys. Rev. B. 77, 075315 (2008).

${ }^{34}$ I. N. Yassievich, A. S. Moskalenko, and A. A. Prokofiev, Mater. Sci. Eng., C 27, 1386 (2007).

${ }^{35}$ K. Huang, and A. Rhys, Proc. R. Soc. A 204, 406 (1950).

${ }^{36}$ H. J. Hrostowski and R. H. Kaiser, Phys. Rev. 107, 966 (1957).

${ }^{37}$ P. Mooney, J. Appl. Phys. 67, R1 (1990).

${ }^{38}$ M. T. Trinh, R. Limpens, W. D. A. M. de Boer, J. M. Schins, L. D. A. Siebbeles, and T. Gregorkiewicz, Nature Photonics (2012), doi:10.1038/nphoton.2012.36. 